\title{
Estrutura fatorial da Stages of Change Readiness and Treatment Eagerness Scale (SOCRATES) em dependentes de álcool tratados ambulatorialmente Factor structure of the Stages of Change Readiness and Treatment Eagerness Scale (SOCRATES) in alcohol dependent outpatients
}

Neliana Buzi Figlie, ${ }^{a}$ John Dunn ${ }^{b}$ e Ronaldo Laranjeira ${ }^{a}$

aUnidade de Pesquisa em Álcool e Drogas (UNIAD), Departamento de Psiquiatria, Universidade Federal de São Paulo, SP, Brasil

broyal Free \& University College Medical School, London

\begin{abstract}
Resumo
objetivo: 0 objetivo deste estudo foi o de investigar a confiabilidade e a estrutura fatorial da Stages of Change Readiness and Treatment Eagerness SCale (SOCRATES), versão 8,1 instrumento com 19 itens que mensura a prontidão para a mudança em dependentes de álcool.

Métodos: Uma análise fatorial confirmatória da SOCRATES foi realizada em uma amostra de 326 dependentes de álcool, tratados ambulatorialmente, tendo como base a estrutura fatorial demonstrada por Miller \& Tonigan ${ }^{2}$ e Maisto et al. ${ }^{3}$

o questionário foi traduzido e adaptado culturalmente para o idioma português, sendo posteriormente submetido ao procedimento da retradução para o idioma inglês. Durante esse procedimento, foram realizadas algumas modificações, visando a simplificar alguns itens que apresentaram formato complexo.

Resultados: As análises estatísticas mostraram a existência de dois fatores correlacionados que melhor exploraram o modelo, sendo este achado similar ao estudo de Maisto et al. ${ }^{3}$

Conclusões: Foi constatada menor evidência para o modelo de três fatores. Esses resultados são comparados com estudos prévios e as discrepâncias são discutidas neste artigo.
\end{abstract}

Descritores: Alcoolismo. Motivação. Análise fatorial. Estudos de validação.

\begin{abstract}
objective: The aim of this study was to investigate the reliability and factor structure of the Stages of Change Readiness and Treatment Eagerness Scale (SOCRATES), version 8, 1 a 19-item self-reported instrument developed to measure readiness to change in alcohol-dependent alcoholics.

Methods: A Confirmatory Factor analysis of the SOCRATES was performed based on the factor structures previously demonstrated by Miller \& Tonigan ${ }^{2}$ and Maisto et al. ${ }^{3}$ in a sample with 326 alcohol-dependent outpatients.

The questionnaire was translated into Portuguese, cross-culturally adapted and back-translated into English. During this process SOCRATES underwent some modifications to simplify some complex question formats.

Results: The analysis showed that two correlated factors provided the best fit for the data and that these were similar to Maisto et al.'s $s^{3}$ factors. Conclusions: There was less evidence to support a three-factor structure. The results are compared to previous studies and the reasons for discrepancies are discussed.
\end{abstract}

Keywords: Alcoholism. Motivation. Factor analysis, statistical. Validation studies.

\section{Introdução}

0 Modelo Trans-Teórico dos Estágios de Mudança (Transtheoretical Model of Stages of Change), descrito por Prochaska e DiClemente, ${ }^{4}$ propõe uma explicação geral e abrangente sobre a forma como as pessoas modificam seus hábitos de dependência, por meio de passos seqüenciais e progressivos (da pré-contemplação à contemplação, determinação, ação e, finalmente, manutenção). Dentro desse marco, a motivação se manifesta por meio de comportamentos específicos aos estágios demonstrados pelos pacientes.

Miller ${ }^{5}$ definiu a motivação como a "probabilidade de uma pes- soa ingressar, continuar e aderir a uma estratégia de mudança específica". A motivação é vista como um estado de prontidão ou avidez para a mudança, que pode flutuar de um momento (ou situação) para outro e pode ser entendido como um estado interno influenciado por fatores externos. 0 conceito de motivação foi extensamente estudado no abuso de substâncias. $6,7,8,5,9$ Em relação ao abuso de álcool, a atribuição do estágio e a prontidão para a mudança do paciente no início do tratamento costumam predizer as medidas de desfecho do tratamento, tais como consumo de álcool e intervalo até a primeira embriaguez. ${ }^{10,11,12}$ 
Miller desenvolveu a Stages of Change Readiness and Treatment Eagerness Scale (SOCRATES) com o objetivo de avaliar a motivação para a mudança em pacientes com consumo problemático de álcool. Uma versão de 32 itens foi inicialmente desenvolvida com seu conteúdo especificamente focado no consumo problemático de álcool. 0 objetivo foi 0 de medir as cinco principais etapas de mudança: pré-contemplação, contemplação, determinação, ação e manutenção.13,14 Desde sua primeira versão, o questionário foi extensamente testado do ponto de vista psicométrico, tendo sido substancialmente modificado em relação ao conteúdo dos itens e ao número total de itens. Sua última versão possui 19 itens e consiste de três fatores ortogonais, que foram denominados Ação, Reconhecimento e Ambivalência. ${ }^{2}$

Maisto et al. ${ }^{3}$ estudaram recentemente a estrutura fatorial da SOCRATES de 19 itens em pacientes de instituições de atendimento primário e encontraram somente dois fatores. 0 primeiro foi denominado AMREC (uma combinação de ambivalência e reconhecimento), com nove itens, e o segundo, Ação, com seis itens e correspondendo ao fator de mesmo nome descrito por Miller \& Tonigan. ${ }^{2}$ Uma análise fatorial confirmatória sugeriu que essa estrutura com dois fatores explorava melhor os dados que a estrutura com três fatores proposta por Miller \& Tonigan. ${ }^{2}$ Outro estudo com adolescentes que se apresentaram para tratamento de transtorno de abuso de álcool identificou dois fatores chamados de ação e reconhecimento. ${ }^{15}$

0 objetivo deste estudo foi o de examinar a confiabilidade e a estrutura fatorial da versão em português da SOCRATES 81 entre pacientes dependentes de álcool e investigar quais das estruturas, de dois ou de três fatores, é a mais adequada aos dados.

\section{Método}

\section{Ambulatórios}

0 estudo foi realizado no Hospital São Paulo/Universidade Federal de São Paulo, um hospital-escola federal. Dois ambulatórios foram utilizados: um de gastroenterologia e outro especializado no tratamento de abuso/dependência de álcool. Consideramos importante termos, na amostra, pacientes que podem apresentar diferentes níveis de motivação em relação à procura de ajuda para seu problema com álcool - daí a escoIha de dois ambulatórios. Os critérios de inclusão foram os seguintes:

1) No ambulatório especializado no tratamento de abuso/dependência de álcool: todos os pacientes que procuraram ajuda para resolver seus problemas relacionados com álcool e que tiveram escores leve, moderado ou grave na ShortForm Alcohol Dependence Data Questionnaire (SADD). ${ }^{16}$ 2) No ambulatório de gastroenterologia: através do AUDIT (The Alcohol Use Disorders Identification Test), ${ }^{17}$ os pacientes que tiveram escores positivos ( $\geq 8)$ foram entrevistados com 0 Short-Form Alcohol Dependence Data Questionnaire (SADD). ${ }^{16}$ Depois disso, os mesmos critérios foram aplicados. Durante o período de estudo, de 21 meses, dos 336 pacientes que se apresentaram no ambulatório de gastroenterologia, 158 (47\%) tiveram escores positivos na AUDIT. ${ }^{17}$
Os critérios de exclusão foram: pacientes que abusaram de outras substâncias, além de álcool, e pacientes que estavam confusos. As mulheres também foram excluídas do estudo. Como havia poucas mulheres apresentando-se em qualquer dos dois serviços, consideramos melhor excluí-las, já que gênero poderia ser um fator de confusão em subseqüentes análises.

\section{Sujeitos}

Foram entrevistados 151 pacientes da clínica gastroenterológica e 175 da clínica especializada de tratamento de abuso/dependência de álcool, totalizando 326 sujeitos. Cinco pacientes recusaram-se a participar do estudo. As entrevistas foram conduzidas nos ambulatórios, na primeira consulta. As características demográficas da amostra estão na Tabela 1 e seus padrões de consumo de álcool e marcadores biológicos estão na Tabela 2.

\section{Medidas}

As entrevistas estruturadas foram conduzidas por três psicólogas experientes. Cartões com opções de resposta foram utilizados para facilitar as respostas dos pacientes. A entrevista consistiu do seguinte:

1) Dados demográficos: idade, gênero, raça, estado civil, escolaridade, ocupação e renda familiar.

2) A Stages of Change Readiness and Treatment Eagerness Scale (SOCRATES), Versão 81: mede os estados de mudança em relação a Reconhecimento, Ambivalência e Ação. A versão 8 possui um sistema de numeração diferente para os itens. Por razões de clareza, utilizamos o sistema de numeração original descrito por Miller \& Tonnigan ${ }^{2}$ para todos os três estudos. 3) Short-Form Alcohol Dependence Data Questionnaire (SADD):

Tabela 1 - Dados sócio-demográficos de 326 homens dependentes de álcool de duas instituições de tratamento ambulatorial

\begin{tabular}{lc|}
\hline \multicolumn{1}{|c}{ CARACTERISTICA } & $\begin{array}{c}\text { PORCENTAGEM } \\
\text { (NÚMERO) }\end{array}$ \\
\hline Idade Média - anos (DP) & $44(11)$ \\
Escolaridade & \\
Analfabeto & $5 \%(16)$ \\
Primeiro grau & $57 \%(187)$ \\
Segundo grau & $25 \%(82)$ \\
Faculdade/Universidade & $13 \%(41)$ \\
& \\
Raça & \\
Branca & $72 \%(235)$ \\
Não-branca (Negro e interracial) & $28 \%(91)$ \\
& \\
Tipo de ocupação & \\
Operacional & $27 \%(89)$ \\
Escritório & $28 \%(90)$ \\
Desempregado & $35 \%(114)$ \\
Outra & $10 \%(33)$ \\
& \\
Renda familiar* & \\
1 a 5 s.m. & \\
5 a 10 s.m. & \\
10 a 20 s.m. & $40 \%(129)$ \\
Acima de 20 s.m. & $32 \%(105)$ \\
Não sabido & $15 \%(51)$ \\
& $9 \%(29)$ \\
Status ocupacional no ano passado & $4 \%(12)$ \\
40 horas semanais & \\
Menos de 40 horas semanais & \\
Desempregado & $49 \%(160)$ \\
\hline
\end{tabular}

NOTA: *s.m. = salário mínimo $=R \$ 151,00$, durante o período de 1998 a 1999. 
Tabela 2 - Padrão de consumo de álcool e marcadores biológicos de dependência de álcool entre homens de duas instituições de tratamento ambulatorial

\begin{tabular}{|c|c|}
\hline VARIÁVEL & $\begin{array}{c}\text { MÉDIA } \\
\text { (VARIAÇÃOINTERQUARTIL) }\end{array}$ \\
\hline Consumo de álcool nos últimos 30 dias - unidades & $81,5(0-281)$ \\
\hline Duração do atual abuso de álcool - semanas & $20(8-96)$ \\
\hline Intervalo desde a última bebedeira - dias & $12(1-42)$ \\
\hline Consumo mensal de álcool durante o uso mais pesado* - unidades & $630(300-1020)$ \\
\hline Duração do período mais pesado de consumo de álcool - semanas* & $240(88-480)$ \\
\hline $\mathrm{GGT}^{\star \star}$ (normal: 11 a $\left.43 \mathrm{U} / \mathrm{L}\right)$ & $87(45-200)$ \\
\hline $\mathrm{AST}^{\star \star *}$ (normal: até $37 \mathrm{U} /$ ) & $39(25-68)$ \\
\hline $\mathrm{ALT}^{* \star \star *}$ (normal: até $\left.40 \mathrm{U} / \mathrm{L}\right)$ & $32(20-59)$ \\
\hline $\begin{array}{l}\text { Gravidade da dependência de álcool - \%(n) } \\
\text { Suave } \\
\text { Moderada } \\
\text { Pesada }\end{array}$ & $\begin{array}{c}19(62) \\
34(112) \\
47(152)\end{array}$ \\
\hline
\end{tabular}

NOTA: Tamanho da amostra ${ }^{\star} n=283,{ }^{\star \star} n=229,{ }^{\star \star \star} n=280,{ }^{\star \star \star \star} n=279$.

esta escala mede a gravidade de dependência de álcool (leve, moderado e grave). A versão em português foi desenvolvida por Jorge e Masur. 16

4) Padrões de consumo de álcool: foram documentados utilizando a escala de entrevista desenvolvida para o WHO/ISBRA Study on State and Trait Markers in Alcoholism. ${ }^{18}$ As perguntas relacionam-se com o padrão de consumo de álcool em relação ao tipo de bebida, à quantidade e à freqüência de uso nos últimos 30 dias, bem como ao período de uso mais pesado de álcool na vida. Uma unidade de álcool foi considerada como equivalente a $10 \mathrm{ml}$ de álcool puro.

5) Marcadores biológicos: aspartato aminotransferase (AST), alanina aminotransferase (ALT) e gama glutamiltransferase (GGT). No ambulatório de gastroenterologia, esses testes eram rotineiros, de forma que a maioria dos pacientes possui resultados; já no ambulatório especializado no abuso/dependência de álcool, um número menor de pacientes apresentou estes resultados, por não se tratar de uma rotina nesse tratamento. Processo de tradução e adaptação cultural da SOCRATES

A escala foi traduzida por dois pesquisadores com um bom domínio de inglês, resultando em duas traduções que foram testadas, cada uma, com um grupo de dez dependentes de álcool, com o objetivo de descobrir qual a forma mais facilmente compreensível para os sujeitos. Esse processo resultou em uma segunda versão, que foi reaplicada em outro grupo de dez alcoolistas, que também foram solicitados a explicar o significado das perguntas e a respondê-las. 0 objetivo foi o de verificar se o paciente e o entrevistador concordavam sobre 0 que as questões realmente significavam. Para cada item, os entrevistados utilizaram uma escala Likert com 5 pontos - variando de 1 (discorda totalmente) a 5 (concorda completamente).

Questões mais complexas tiveram que ser simplificadas, pois os pacientes tendiam a responder somente parte da questão, sem referir-se à outra metade. Por exemplo, a questão 20: "Eu tenho feito algumas mudanças no meu consumo de álcool e quero alguma ajuda para não voltar a beber como antes". 0s pacientes tenderam a responder a esta questão concordando ou discordando que tinham feito mudanças na sua forma de beber ou que tinham desejado ajuda para impedi-los de voltar a beber como antes. Para complicar mais esse problema, se os pacientes não tivessem realizado nenhuma mudança em sua forma de beber, então, por definição, eles não poderiam responder à pergunta. Nossa solução a esse problema foi dividir a pergunta em seus componentes cronológicos e seqüenciais, primeiro: "Você fez mudanças no seu hábito de beber?". Se os pacientes respondessem negativamente, seriam automaticamente atribuídos à categoria discordo muito. Se respondessem afirmativamente, o segundo componente da pergunta seria colocado para eles: "Eu quero alguma ajuda para não voltar a beber como antes", para o qual eles pode- riam escolher entre discordo muito a concordo muito. Pode-se argumentar que isso não é uma solução satisfatória, já que os pacientes podem ainda querer parar de beber, mesmo que não desejem o tipo de ajuda declarado na pergunta. Em vez de excluir totalmente essas três perguntas, sentimos que seria melhor o meio termo. 0 mesmo procedimento foi seguido para as perguntas 14 e 15. Por outro lado, é importante notar que esse processo pode prejudicar a comparação entre os resultados obtidos pela versão original e a versão em português.

A terceira versão do questionário foi, então, submetida a um comitê ad hoc, composto por um psicólogo brasileiro - que trabalhava na área de dependência de drogas e que tinha um bom entendimento de inglês -, um profissional brasileiro que trabaIhava com dependência de drogas e não falava inglês, um psiquiatra britânico que trabalhava com dependência de drogas e era fluente em português e um psicólogo britânico que trabalhava com dependência de drogas e tinha algum conhecimento de português. 0 comitê estudou cada pergunta, verificando o original em inglês e suas várias traduções em português. Ao final, um consenso foi alcançado em relação a qual versão era mais facilmente compreensível pelo paciente brasileiro médio, ao mesmo tempo em que mantinha o conceito original da pergunta.

A versão do questionário feita pelo comitê ad hoc foi aplicada a outro grupo de pacientes com dependência de álcool, de 
forma que cada pergunta fosse feita a cinco sujeitos diferentes Para os pacientes, o objetivo era de que eles dessem seu entendimento sobre 0 que as perguntas significavam e as respondessem. Essas entrevistas foram gravadas. Pequenas modificações foram feitas após cada entrevista antes da versão final. ${ }^{19} \mathrm{~A}$ versão em português da forma final está no Anexo 1. A tradução reversa foi feita por um professor de inglês americano, sem referenciar-se na versão original do questionário.

\section{Considerações éticas}

0 estudo foi aprovado pelo Comitê de Ética para Pesquisas Médicas da Universidade Federal de São Paulo, Brasil. Todos os sujeitos receberam informações sobre a pesquisa, tendo assinado 0 formulário do consentimento informado antes de participar e assegurados 0 anonimato e a confidencialidade.

\section{Análise estatística}

0 primeiro passo foi investigar a estrutura fatorial e a validade de construto do questionário de 19 itens da SOCRATES. Isso foi feito utilizando duas análises fatoriais de confirmação de primeira ordem, a primeira modelada sobre as três estruturas fatoriais descritas por Miller \& Tonigan ${ }^{2}$ e a segunda sobre os dois fatores independentes, descrita por Maisto et al. ${ }^{3} \mathrm{~A}$ confiabilidade dos fatores foi medida pelo exame de sua consistência interna - usando o alfa de Cronbach - e a análise de itens foi baseada na correlação entre os itens e o escore total. A correlação entre os fatores e os resultados das análises de itens sugere a existência de uma estrutura fatorial baseada em dois fatores não-independentes.
Utilizando o pacote estatístico SAS, a covariância gerada pela CALIS (Covariance Analysis of Linear Structural Equations) foi utilizada para testar os modelos de análise fatorial confirmatória, estimar os parâmetros e testar a adequação dos modelos de equações estruturais utilizando análise estrutural de covariância e estimativa de probabilidade máxima. 0 ajustamento dos diferentes modelos foi avaliado por meio de quatro índices: 1) o Goodness Fit Index (GFI), com variação de 0 a 1 , sendo 0,90 a maior evidência de um modelo com bom ajustamento;20 2) o Adjusted Goodness-of-Fit Indices (AGFI), que se refere a um GFI ajustado para graus de liberdade no modelo, também variando entre 0 e 1;21 3) uma razão entre o qui-quadrado e os graus de liberdade ( $X^{2} / \mathrm{df}$ ) menor que $2 ; 22$ 4) raiz quadrada do erro quadrático médio (RMSR) menor que 0,05;22 e 5) um erro de aproximação da raiz quadrática média (RMSEA) de menos de $0,08.23$

\section{Resultados}

Análise fatorial confirmatória: modelos de dois e três fatores

Para avaliar a estrutura de três fatores, proposta por Miller \& Tonigan, ${ }^{2}$ e a de dois fatores, proposta por Maisto et al., ${ }^{3}$ utilizamos a análise fatorial confirmatória (AFC) baseada nesses modelos, com erros e fatores independentes.

Para o modelo de três fatores, todos os itens têm peso significativo nas dimensões correspondentes - como se pode ver na Tabela 3. 0 qui-quadrado desse modelo foi de 850,25 , g.l. $=152$, $\mathrm{p}<0,0001$. Os índices de ajustamento foram: $\mathrm{GFI}=0,793$, AGFI $=0,7417, \mathrm{RMSR}=0,203$ e RMSEA $=0,1187$, o que mostra um

Tabela 3 - Análise Fatorial Confirmatória da escala SOCRATES, com carga fatorial e análise de confiabilidade usando o alfa de Cronbach deste estudo baseado na estrutura de três fatores proposta por Miller \& Tonigan (1996) e na estrutura de dois fatores Cronbach deste estudo baseado
proposta por Maisto et al. -1999

\begin{tabular}{|c|c|c|c|c|c|c|c|c|c|c|c|}
\hline \multirow[t]{4}{*}{ ITENS } & \multicolumn{5}{|c|}{$\begin{array}{l}\text { ANÁLISE FATORIAL CONFIRMATÓRIA } \\
\text { (PESOS DOS FATORES) }\end{array}$} & \multirow[t]{4}{*}{ ITENS } & \multicolumn{5}{|c|}{$\begin{array}{l}\text { ANÁLISE DE CONFIABILIDADE } \\
\text { (ALFADE CHRONBACH) }\end{array}$} \\
\hline & \multicolumn{3}{|c|}{ Modelo de Miller \& Tonigan (1996) } & \multicolumn{2}{|c|}{$\begin{array}{l}\text { Modelo de Maisto } \\
\text { et al. (1999) }\end{array}$} & & \multicolumn{3}{|c|}{ Modelo de Miller \& Tonigan (1996) } & \multicolumn{2}{|c|}{$\begin{array}{l}\text { Modelo de Maisto } \\
\text { et al. (1999) }\end{array}$} \\
\hline & Fator 1 & Fator 2 & Fator 3 & Fator 1 & Fator 2 & & Fator 1 & Fator 2 & Fator 3 & Fator 1 & Fator 2 \\
\hline & Reconhecimento & Ambivalência & Ação & AMREC & Ação & & Reconhecimento & Ambivalência & Ação & AMREC & Ação \\
\hline 1 & $0,212^{*}$ & & & - & & 1 & 0,214 & & & - & \\
\hline 3 & $0,574^{\star}$ & & & $0,592^{*}$ & & 3 & 0,551 & & & 0,554 & \\
\hline 8 & $0,803^{*}$ & & & $0,801^{*}$ & & 8 & 0,730 & & & 0,720 & \\
\hline 11 & $0,863^{*}$ & & & $0,845^{*}$ & & 11 & 0,747 & & & 0,736 & \\
\hline 13 & $0,834^{*}$ & & & $0,828^{*}$ & & 13 & 0,732 & & & 0,738 & \\
\hline 16 & $0,663^{*}$ & & & $0,667^{\star}$ & & 16 & 0,610 & & & 0,602 & \\
\hline 18 & $0,574^{*}$ & & & $0,597^{\star}$ & & 18 & 0,513 & & & 0,564 & \\
\hline 2 & & $0,648^{*}$ & & $0,600^{\star}$ & & 2 & & 0,509 & & 0,614 & \\
\hline 7 & & $0,678^{*}$ & & $0,553^{*}$ & & 7 & & 0,510 & & 0,539 & \\
\hline 12 & & $0,239^{*}$ & & $0,214^{\star}$ & & 12 & & 0,211 & & 0,210 & \\
\hline 17 & & $0,718^{*}$ & & - & & 17 & & 0,510 & & - & \\
\hline 4 & & & $0,685^{\star}$ & & $0,685^{\star}$ & 4 & & & 0,526 & & 0,574 \\
\hline 5 & & & $0,507^{\star}$ & & $0,528^{*}$ & 5 & & & 0,326 & & 0,415 \\
\hline 9 & & & $0,554^{*}$ & & $0,574^{*}$ & 9 & & & 0,389 & & 0,424 \\
\hline 10 & & & $0,721^{*}$ & & $0,724^{*}$ & 10 & & & 0,524 & & 0,616 \\
\hline 14 & & & $0,396^{\star}$ & & $0,336^{\star}$ & 14 & & & 0,378 & & 0,282 \\
\hline 15 & & & $0,169^{*}$ & & - & 15 & & & 0,174 & & - \\
\hline 19 & & & $0,646^{\star}$ & & $0,646^{*}$ & 19 & & & 0,503 & & 0,536 \\
\hline \multirow[t]{2}{*}{20} & & & $0,472^{*}$ & & - & 20 & & & 0,450 & & - \\
\hline & & & & & & Alfa & 0,828 & 0,646 & 0,679 & 0,856 & 0,714 \\
\hline
\end{tabular}

NOTA: *O coeficiente (pesos de fatores) é significativo no nivel 0,01 (teste bicaudado). 


\begin{tabular}{|c|c|c|c|c|c|c|}
\hline \multirow{3}{*}{$\begin{array}{l}\text { Miller \& Tonigan } \\
\text { (1996) }\end{array}$} & \multirow[b]{3}{*}{ Reconhecimento } & \multicolumn{3}{|c|}{ MILLER \& TONIGAN (1996) } & \multicolumn{2}{|c|}{ MAISTO ET AL. (1999) } \\
\hline & & Reconhecimento & Ambivalência & Ação & AMREC & Ação \\
\hline & & 1,00 & $0,72^{\star}$ & $0,39^{*}$ & $0,96^{*}$ & $0,28^{*}$ \\
\hline & $\begin{array}{l}\text { Ambivalência } \\
\text { Ação }\end{array}$ & $\begin{array}{l}0,72^{\star} \\
0,39^{\star}\end{array}$ & $\begin{array}{l}1,00 \\
0,28^{\star}\end{array}$ & $\begin{array}{c}0,28^{*} \\
1,00\end{array}$ & $\begin{array}{l}0,87^{\star} \\
0,36^{*}\end{array}$ & $\begin{array}{l}0,24^{\star} \\
0,89^{\star}\end{array}$ \\
\hline \multirow{2}{*}{$\begin{array}{l}\text { Maisto et al. } \\
\text { (1999) }\end{array}$} & AMREC & $0,96^{*}$ & $0,87^{\star}$ & $0,36^{*}$ & 1,00 & $0,26^{*}$ \\
\hline & Ação & $0,28^{*}$ & $0,24^{*}$ & $0,89^{*}$ & $0,26^{*}$ & 1,00 \\
\hline
\end{tabular}

NOTA: *A correlação é significativa no nível 0,01 (bicaudado).

ajustamento menor que o ideal entre o modelo de três fatores proposto e os dados atuais.

Para o modelo de dois fatores (AMREC: AmbivalênciaReconhecimento e Ação), todos os itens tinham peso significativo nas dimensões correspondentes (Tabela 3). 0 qui-quadrado desse modelo foi de 335,72 , g.I. $=90, p<0,0001$. 0 s índices de ajustamento foram: $\mathrm{GFI}=0,8866, \mathrm{AGFl}=0,8488, \mathrm{RMSR}=0,1351 \mathrm{e}$ RMSEA $=0,0915$. Todos esses índices sugerem um melhor ajustamento para o modelo de dois fatores, como foi proposto por Maisto et al. ${ }^{3}$

Confiabilidade e análise de itens dos modelos de dois e três fatores

Para o modelo de três fatores, de Miller \& Tonigan, ${ }^{2}$ os itens que compõem o fator 1 (Reconhecimento) possuem uma boa confiabilidade, com correlações item-total acima de 0,51 - exceto 0 item 1. Para o fator 2 (Ambivalência), as correlações são maiores que 0,50 - exceto 0 item 12. Para o fator 3 (Ação), as correlações são ainda menores, mas acima de 0,3 - exceto 0 item 15. Para o modelo de dois fatores, de Maisto, ${ }^{3}$ o fator 1 (AMREC) possui boa confiabilidade com o item para correlações acima de 0,54 - exceto o item 12. 0s itens no fator 2 (Ação) possuem correlações maiores que 0,41 - exceto o item 14. Esses resultados sugerem que os itens com baixas correlações devem ser excluídos da análise (Tabela 3).

\section{Correlação dos fatores}

Para explorar a relação entre os fatores baseando-se nos dois modelos, estudamos as correlações intra e interfatores. As correlações intrafatores mostram se os fatores dentro de cada modelo são independentes um do outro. As correlações interfatores mostram a relação entre os dois modelos. Para o modelo de três fatores, há uma forte correlação entre Reconhecimento e Ambivalência. Para o modelo de dois fatores, as correlações são muito menores (Tabela 4).

As correlações interfatores entre os dois modelos mostram que Reconhecimento e Ambivalência estão estreitamente correlacionados com o AMREC, como o estão os fatores Ação de cada modelo.

Esses resultados sugerem que os três fatores descritos por Miller \& Tonigan² e os dois fatores (AMREC e Ação) propostos por Maisto et al. ${ }^{3}$ não são independentes, indicando que uma nova estrutura fatorial deve ser investigada.

Análise de itens, confiabilidade e AFC para um modelo de dois fatores correlacionados

Uma análise de itens e o alfa de Cronbach foram utilizados com os dados modelados nos primeiros e segundos fatores combinados, conforme descrição de Maisto et al. ${ }^{3} 0$ alfa de Cronbach foi de 0,846 e houve baixas correlações para os itens $1(0,259)$

\begin{tabular}{|c|c|c|c|c|}
\hline \multirow[b]{2}{*}{ Itens } & \multicolumn{2}{|c|}{$\begin{array}{l}\text { PRIMEIRA ANÁLISE COM } \\
\text { TODOS OS ITENS }\end{array}$} & \multicolumn{2}{|c|}{$\begin{array}{l}\text { SEGUNDAANÁLISE EXCLUINDOITENS COM } \\
\text { BAIXAS CORRELAÇÕES }\end{array}$} \\
\hline & Fator 1 & Fator 2 & Fator 1 corrigido & Fator 2 corrigido \\
\hline 1 & 0,259 & & - & \\
\hline 3 & 0,574 & & 0,565 & \\
\hline 8 & 0,710 & & 0,724 & \\
\hline 11 & 0,723 & & 0,739 & \\
\hline 13 & 0,722 & & 0,747 & \\
\hline 16 & 0,609 & & 0,635 & \\
\hline 18 & 0,571 & & 0,595 & \\
\hline 2 & 0,617 & & 0,614 & \\
\hline 7 & 0,567 & & 0,554 & \\
\hline 12 & 0,227 & & - & \\
\hline 17 & 0,659 & & 0,681 & \\
\hline 4 & & 0,526 & & 0,562 \\
\hline 5 & & 0,326 & & 0,369 \\
\hline 9 & & 0,389 & & 0,383 \\
\hline 10 & & 0,524 & & 0,598 \\
\hline 14 & & 0,378 & & 0,420 \\
\hline 15 & & 0,174 & & - \\
\hline 19 & & 0,503 & & 0,521 \\
\hline 20 & & 0,450 & & 0,476 \\
\hline Alfa & 0,865 & 0,679 & 0,890 & 0,738 \\
\hline
\end{tabular}




\begin{tabular}{|c|c|c|}
\hline $\begin{array}{c}\text { VARIÁVEIS OBSERVADAS } \\
\text { (ITENS) }\end{array}$ & $\begin{array}{l}\text { FATOR1 } \\
\text { CORRIGIDO }\end{array}$ & FATOR 2 CORRIGIDO \\
\hline 3 & $0,601^{\star}$ & \\
\hline 8 & $0,791^{\star}$ & \\
\hline 11 & $0,826^{*}$ & \\
\hline 13 & $0,822^{\star}$ & \\
\hline 16 & $0,682^{*}$ & \\
\hline 18 & $0,615^{\star}$ & \\
\hline 2 & $0,609^{\star}$ & \\
\hline $\begin{array}{l}2 \\
7\end{array}$ & $0,576^{\star}$ & \\
\hline 12 & Não analisado & \\
\hline 17 & $0,690^{\star}$ & \\
\hline 4 & & $0,694^{\star}$ \\
\hline 5 & & $0,514^{\star}$ \\
\hline 9 & & $0,568^{\star}$ \\
\hline 10 & & $0,698^{\star}$ \\
\hline 14 & & $0,360^{\star}$ \\
\hline 15 & & não analisado \\
\hline 19 & & $0,669^{\star}$ \\
\hline 20 & & $0,454^{\star}$ \\
\hline
\end{tabular}

NOTA: *O coeficiente (pesos fatoriais) é significativo no nivel 0,01 (teste bicaudado)

e $12(0,2274)$, mostrando que esses itens não são parte do fator. Esses itens foram excluídos e os dados foram reanalisados. 0 fator 1, corrigido, tem 9 itens e é muito similar ao fator AMREC de Maisto et al., 3 exceto pelo fato de que incorporou o item $17 \mathrm{e}$ excluiu 0 item 12. Sua correlação item-total foi maior que 0,55 e seu alfa foi de 0,89. 0 item 15 foi então excluído do fator 2. 0 fator 2, corrigido, tem 7 itens e é similar ao segundo fator de Maisto et al., ${ }^{3}$ exceto pelo fato de que incorpora 0 item 20. Nós denominamos nosso primeiro fator como AmRec e o segundo, como Ação.

Para avaliar a estrutura desses dois fatores não-independentes, foi realizado outra AFC. A hipótese da existência dessa nova estrutura fatorial de dois fatores é parcialmente confirmada pelos resultados da análise. Todos os itens possuem peso significativo $(p<0,001)$ nas dimensões correspondentes (como se pode visualizar na Tabela 6). 0 qui-quadrado desse modelo foi de 407,22 (g.I.=103, $p<0,0001$ ) - razão entre $X^{2}$ e g.I = $407,22 / 103>2$-, que mostra que existem diferenças entre 0 modelo proposto e os dados. 0s índices de ajustamento foram: $\mathrm{GFI}=0,869, \mathrm{AGFI}=0,827, \mathrm{RMSR}=0,091$ e RMSEA $=0,0856$. Esses resultados mostram que o modelo demonstra um ajustamento bom, porém imperfeito. A correlação entre os fatores foi de $0,364(p<0,01), 0$ que é estatisticamente significativo.

\section{Correlação com o consumo total de álcool nos últimos 30 dias}

Para examinar a validade dos diferentes modelos, calculamos a correlação entre os escores obtidos em cada fator com o consumo total de álcool nos 30 dias anteriores à pesquisa. Como essa medida teve uma distribuição assimétrica, foi feita uma transformação logarítmica. Tendo sido nossos dados modelados pela estrutura de três fatores, de Miller \& Tonigan, 2 as correlações de Pearson foram as seguintes: Ambivalência ( $r=0,240$, $p<0,001)$, Reconhecimento ( $r=0,211, p<0,001)$ e Ação $(r=-0,178$, $p<0,001)$. Para o modelo baseado no modelo de dois fatores, de Maisto et al., ${ }^{3}$ as correlações foram: AMREC $(r=0,219, p<0,001)$ e Ação $(r=-0,142, p<0,01)$.

\section{Discussão}

0 Modelo Trans-Teórico de Estágios de Mudança foi questionado e as tentativas de medir os cinco estágios como entidades distintas tiveram êxito limitado. 24,25,26,27 0 objetivo deste artigo foi 0 de investigar a estrutura fatorial da versão em português da escala SOCRATES. Miller \& Tonigan $^{2}$ afirmam que a escala SOCRATES possui uma estrutura de três fatores, que consiste de Ambivalência, Reconhecimento e Ação. Encontramos que uma estrutura de dois fatores não-independentes ajusta-se melhor aos dados do que uma de três fatores. 0s dois fatores estavam compostos de itens agrupados ao redor de dois diferentes conceitos, denominados de AmRec (uma combinação de Ambivalência e Reconhecimento) e Ação, similares aos resultados obtidos por Maisto et al. ${ }^{3}$

Um estudo com estudantes universitários ${ }^{28}$ deu um modesto apoio para que a escala SOCRATES tivesse uma estrutura de três fatores. Nesse estudo, os autores utilizaram uma análise fatorial confirmatória com um questionário SOCRATES com 19 itens e encontraram que o modelo de três fatores ajusta-se aos dados de forma moderadamente boa. A seguir, eles removeram os três itens com as cargas fatoriais cruzadas (cross loadings) mais fracas e repetiram a análise. A versão com 16 itens teve melhor desempenho que a versão completa.

Maisto et al.3,15 encontraram dois fatores que eram quase iguais aos nossos. Esses autores citam trabalho não-publicado que sugere que o fator Ambivalência, encontrado por Miller \& Tonigan (1996), pode ser instável. Eles citam que Dermen et al. ${ }^{29}$ não encontraram cargas fatoriais cruzadas significativas para os itens de Ambivalência na escala SOCRATES em uma amostra de pacientes de um centro de internação para tratamento de abuso de substâncias e que Busby \& Parker ${ }^{30}$ encontraram que a escala de Ambivalência tinha consistência interna pobre.

Há variadas razões para a discrepância entre a estrutura fatorial relatada por Miller \& Tonigan² e a encontrada nos estudos subseqüentes. Primeiro, o instrumento, no seu formato atual, pode não ser capaz de distinguir entre Ambivalência e Reconhecimento, porque o conteúdo dos itens do questionário é inadequado para realizar tal distinção. Nosso processo de tradução identificou ambigüidade em vários itens e os sujeitos relataram que alguns itens foram difíceis de serem respondidos. Com perguntas melhores e mais precisas para caracterizar Ambivalência, poderia ser possível refinar e modificar 0 instrumento para fazer uma distinção mais clara entre esse fator e o Reconhecimento.

Uma segunda explicação é que a Ambivalência e o Reconhecimento são, de fato, parte do mesmo fenômeno. A partir da experiência clínica cotidiana, pode-se imaginar pacientes que reconheçam terem problemas com álcool, mas que sejam ambivalentes quanto à necessidade de pararem de beber para modificarem seus estilos de vida ou para seguir um programa de tratamento prescrito. Em estágios precoces do tratamento, a Ambivalência e o Reconhecimento são, em geral, observadas 
conjuntamente. Nossa análise fatorial sugere que há substancialmente mais semelhança entre esses conceitos do que um caráter único, já que o conjunto de perguntas desenhadas para medi-los não varia independentemente, mas, de fato, está altamente correlacionado a um único fator.

Maisto et al. ${ }^{3}$ propuseram uma explicação alternativa para seu insucesso em reproduzir a solução de três fatores de Miller \& Tonigan, ${ }^{2}$ em particular por não terem encontrado um fator de Ambivalência. No estudo de Miller \& Tonigan, ${ }^{2}$ os pacientes provinham de serviços de atenção secundária e tinham níveis consideravelmente mais altos de consumo de álcool e uma gravidade do problema de uma natureza e grau que seria facilmente reconhecível pela maioria dos observadores. Eles sugerem que, se os indivíduos ainda estão incertos quanto a terem problemas com a bebida (dependência de álcool e complicações secundárias), então outros fatores podem estar em jogo (tais como a negação patológica ou o déficit cognitivo), o que poderia refletir-se em um fator de ambivalência. No entanto, nossa amostra também deriva de serviços de tratamento secundário - a maioria dos pacientes era moderada ou gravemente dependente e aqueles provindos do ambulatório de gastroenterologia já tinham desenvolvido complicações físicas do consumo de álcool -, mas, mesmo assim, fomos incapazes de encontrar evidências convincentes de um fator de ambivalência separado.

É possível que esses diferentes achados devam-se, em parte, aos diferentes procedimentos de análise fatorial. 0 estudo de Miller \& Tonigan ${ }^{2}$ utilizou uma análise fatorial com análise de componentes principais com rotações ortogonais e não-ortogonais e um critério de eingenvalues $>1,0$. Maisto et al. ${ }^{3}$ utilizaram uma análise de componentes principais com rotação ortogonal para extrair fatores com eigenvalues $\geq 1,0$, seguida por uma análise fatorial confirmatória com rotação ortogonal. Também utilizaram uma análise da excelência de ajustamento para comparar seu modelo de dois fatores com a solução de três fatores de Miller \& Tonigan $^{2}$ e encontraram que a solução de dois fatores ajustava-se melhor aos dados.

A remoção dos três itens de baixa confiabilidade (perguntas 1, 12 e 15) pode ter tido algum efeito em nossa análise fatorial Miller \& Tonigan ${ }^{2}$ utilizaram a escala SOCRATES de 20 itens, mas eliminaram um deles (pergunta 6) devido à sua baixa carga fatorial e à dificuldade de fazer a pergunta em uma instituição de acompanhamento clínico. Maisto ${ }^{3}$ excluiu o item 17 devido a um erro de cálculo. Eles então excluíram os itens 1, 15 e 20. 0s três itens que nós removemos foram os das seções Ação e Reconhecimento do questionário.

Nosso estudo utilizou uma versão em português da escala SOCRATES, portanto, o processo de tradução deve ser examinado, pois pode ter afetado também o desempenho do instrumento. Uma má tradução pode afetar a consistência interna, a confiabilidade e a validade de um instrumento desenvolvido em outro idioma e outra cultura. ${ }^{31,32}$ Seguimos um meticuloso processo para assegurar que uma versão semanticamente equivalente, confiável e válida fosse produzida. Isso envolveu traduções alternativas do questionário produzido e um comitê bilíngüe multiprofissional, que examinou cada pergunta, gravou as entrevistas com os pacientes e realizou um extenso teste- piloto. 0s pacientes, por sua vez, não somente eram solicitados a responder às perguntas, mas também a dizerem o que elas significavam. A análise psicométrica demonstrou que nossa versão possuía boa consistência interna e confiabilidade. Nosso modelo de dois fatores é consistente com os achados de outros estudos, ainda que, dos três itens excluídos devido às suas baixas correlações inter-itens, somente um tenha sido excluído por Maisto et al. ${ }^{3}$ (item 1). Recorremos à alteração da estrutura de três perguntas, o que poderia ter mudado o desempenho do questionário. Percebemos que essas modificações eram necessárias para possibilitar que todos os pacientes respondessem a todas as perguntas, mas nossa solução não foi inteiramente satisfatória.

Sutton ${ }^{26}$ questionou os achados de Miller \& Tonigan ${ }^{2}$ e os criticou por não relatarem a matriz de correlação completa. Notou que as sub-escalas de pré-contemplação e determinação estiveram negativamente correlacionadas $(-0,70$ na amostra de pacientes hospitalizados e -0,62 na amostra de pós-tratamento), que as sub-escalas Ação e Manutenção estavam positivamente correlacionadas $(0,69$ e 0,56$)$, e que outras correlações foram modestas. Ele interpretou esses resultados como uma sugestão de falta de distinção entre pré-contemplação e determinação e entre ação e manutenção.

\section{Conclusão}

São necessários mais estudos para investigar a estrutura fatorial da escala SOCRATES em diferentes populações clínicas e culturais, em especial fora dos países anglo-saxões. Mais estudos são necessários para entender os aspectos que a escala SOCRATES não mede e isso demonstra que essa escala possa ser melhorada.

\section{Agradecimentos}

Os autores gostariam de agradecer à Janaina Turisco e Roberta Paya por sua ajuda com a coleta de dados e às equipes dos ambulatórios que tornaram este estudo possível.
Financiamento: Fundação de Amparo à Pesquisa do Estado de São Paulo - FAPESP. Recebido em 21.10.2003 Aceito em 04.07.2003 
Anexo 1 - Versão em português da escala Stages of Change Readiness and Treatment Eagerness Scale (SOCRATES)

DISCORDO DISCORDO INDECISO CONCORDO CONCORDO

1- Eu realmente gostaria de fazer MUITO MUITO

mudanças na minha maneira de beber.

2- As vezes, eu penso se sou um alcoólatra.

3- Se eu não mudar logo a minha maneira de beber, meus problemas vão ficar piores.

4- Eu já comecei a fazer algumas mudanças em relação à minha maneira de beber.

5- Estou tentando controlar a minha maneira de beber.

6- As vezes, eu penso se o meu beber está prejudicando as outras pessoas.

7- Eu sou uma pessoa que tem problemas com bebidas alcoólicas.

8- Eu não estou pensando apenas nas mudanças da minha maneira de beber. Eu já estou fazendo alguma coisa sobre isso.

9- Eu já mudei o meu hábito de beber estou buscando meios para não voltar a beber como bebia antes.

10- Eu tenho graves problemas com a bebida.

11- As vezes, eu penso se tenho controle sobre a minha maneira de beber.

12- O meu hábito de beber esta causando muitos transtornos.

13- Você parou ou diminui seu hábito de beber?

( )Não - Coloque a resposta Discordo Muito e vá para Q14.

( ) Sim - Atualmente estou fazendo coisas para diminuir ou parar o meu beber.

14- Antes você tinha problemas com a bebida?

()Não - Coloque a resposta Discordo Muito e vá para Q15.

()Sim - Eu quero ajuda para evitar que eu volte a ter os problemas com a bebida que eu tinha antes.

15- Eu sei que tenho problemas com bebidas alcoólicas.

16- As vezes, eu acho que bebo demais.

17- Eu sou um alcoólatra.

18- Eu estou me esforçando muito para mudar o meu hábito de beber.

19- Você fez mudanças no seu hábito de beber?

()Não - Coloque a resposta Discordo

Muito.

( )Sim - Eu quero alguma ajuda para não voltar a beber como antes. 


\section{Referências}

1. Miller WR. SOCRATES The Stages of Change Readiness and Treatment Eagerness Scale (Version 8). Albuquerque (NM): Univ New Mexico; 1995

2. Miller WR, Tonigan JS. Assessing Drinkers' Motivation for change: The Stages of Change Readiness and Treatment Eagerness Scale (SOCRATES). Psychiatry Addict Behav 1996;10(2):81-9.

3. Maisto AS, Conigliaro J, McNeil M, Kraemer K, O'Connor M, Kelley ME. Factor structure in a sample of primary care patients. Addict Behav 1999; 24(6): 879-92.

4. DiClemente CC, Prochaska JO, Velicer WF, Fairhurst S, Rossi JS, Velaquez M. The process of smoking cessation: an analysis of the precontemplation, contemplation and preparation stages of change. $J$ Cons Clin Psychology 1991;59:295-304.

5. Miller WR. Motivation for treatment: a review with special emphasis on alcoholism. Psychological Bull 1985;98:84-107.

6. Connors GJ, Donovan DM, DiClemente CC. Substance Abuse Treatment and Stages of Change. New York (NY): The Guilford Press; 2001.

7. Tucker JA, Donovan DM, Marlatt GA. Changing Addictive Behavior Bridging Clinical and Public Health Strategies. New York: The Guilford Press; 1999 .

8. Miller WR, Rollnick S. Motivational Interviewing - preparing people to change addictive behaviour. New York: The Guilford Press; 1991.

9. Miller WR. Motivational interviewing with problem drinkers. Behav Psychotherapy 1983:11:147-72.

10. Heather N, Rollnick S, Bell A. Predictive validity of the Readiness to Change Questionnaire. Addiction 1993;88:1667-77.

11. Buddy R, Rollnick S. (1996) The structure of the Readiness to Change questionnaire: a test of Prochaska and DiClemente's transtheoretical model. Br J of Health Psychology 1994;1:365-76.

12. McMahon J, Jones BT. Post-treatment abstinence survivorship and motivation for recovery: the predictive validity of the readiness to change (RCQ) and negative alcohol expectancy (NAEQ) questionnaires. Addiction Research 1996;4:161-76.

13. Miller WR, Meyers RJ, Hester RK., Delaney H, Montgomery HA, Abbott $P J$. The effectiveness of the community reinforcement approach: Preliminary findings of a clinical trial. Paper presented at the $5^{\text {th }}$ international conference on Treatment of Addictive Behaviours; 1990; Sydney, Australia.

14. Miller WR, Tonigan JS, Montgomery HA, Abbott PJ, Meyers RJ, Hester $R K$, Delaney $H$. Assessment of client motivation for change: preliminary validation of the SOCRATES instrument. Paper presented at the annual meeting of the Association for Advancement of Behavior Therapy; 1990; San Francisco, CA.

15. Maisto SA, Chung TA, Cornelius JR, Martin CS. Factor structure of the SOCRATES in a clinical sample of adolescents. Psychology Addict Behav 2003:17(2):98-107.

16. Jorge MR, Masur J. The use of the Short-Form Alcohol Dependence Data Questionnaire (SADD) in Brazilian Alcoholic Patients. $\mathrm{Br} J$ Addiction 1985;80:301-5.

17. Méndez EB. A Brazilian version of the AUDIT (Alcohol Use Disorders Identification Test). Unpublished master dissertation. Universidade Federal de Pelotas - Social Medicine Department Post-Graduate Program; 1999

18. Tabakoff B, Dongier M. The WHO/ISBRA Study on State and Trait Markers in Alcoholism: Progress Report. Alcohol Clin Exp Research 1996;20(8):23-7.

19. Figlie, NB. Motivation in alcoholic outpatient in specialised alcoholism and gastroenterology clinical treatment program. Unpublished master dissertation. Universidade Federal de São Paulo - Psychiatry Post-Graduate Program; 1999.
20. Byrne BM. Structural Equation Modelling with EQS and EQS/Windows: Basic Concepts, Applications, and Programming. Thousand Oaks (CA): Sage; 1994.

21. Pedhazur EJ, Schmelkin LP. Measurement, Design and Analysis: An integrated approach. New Jersey (NJ): Lawrence Erlbaum Associates Inc., Publishers; 1991.

22. Tabachnick BG, Fidell LS. Using Multivariate Statistics. $3^{\text {rd }}$ ed. New York: Harper Collins; 1996.

23. Arbuckle JL. Amos User's Guide (Version 3.6). Chicago (IL): Smallwaters Corporation; 1997.

24. Davidson R. Editorial and commentaries: "Prochaska and Diclemente's model of change: a case-study?" $\mathrm{Br}$ Jf Addiction 1992;87:21-835.

25. Sutton S. Can "stages of change" provide guidance in the treatment of addictions? A critical examination of Prochaska and DiClemente's model. In: Psychological Treatments and the Addictions. Cambridge (UK): Cambridge University Press; 1996. p. 189-205.

26. Sutton S. Back to the drawing board? A review of applications of the transtheoretical model to substance use. Addiction 2001;96:175-86. 27. Sutton S. Assessing stage of change in current and former smokers. Addiction 2002;97:1171-82.

28. Vik PW, Culbertson KA, Sellers K. Readiness to change among heavydrinking college students. J Stud Alcohol 2000;61:674-80.

29. Dermen K, Kousty J, Connors GJ, Czarnecki D. SOCRATES scores of alcoholic patients: Factor structure and relationship to pretreatment characteristics. Paper presented at the Annual Meeting of the Association for the Advancement of Behavior Therapy; 1997; Miami, FL. 30. Busby $L D$, Parker JD. Norms and reliability for the stages of change and treatment eagerness scale (SOCRATES) obtained from drinkers accepted for inpatient treatment. Poster session presented at the Annual Meeting of the Association for the Advancement of Behavior Therapy; 1997; Miami, FL.

31. Perneger TV, Leplege A, Etter JF. Cross-cultural adaptation of a psychometric instrument: two methods compared. I Clin Epidem 1999:52(11): 1037-46.

32. Lee JA, More SJ, Cotiwan BS. Problems of translating a questionnaire in a cross-cultural setting. Prev Vet Medicine 1999;41:187-94.

Correspondência Neliana Buzi Figlie Unidade de Pesquisa em Álcool e Drogas (UNIAD), UNIFESP/EPM

Departamento de Psiquiatria Rua Borges Lagoa, 564 - cj. 44 04038-000 São Paulo, SP, Brasil

Tel./Fax: (55 11) 5579-0640

Cel.: (55 11) 9659-3327 E-mail: nelianafiglie@uol.com.br e neliana@psiquiatria.epm.br 